\title{
Erratum to: Black hole solution and strong gravitational lensing in Eddington-inspired Born-Infeld gravity
}

\author{
Shao-Wen Wei ${ }^{\mathrm{a}}$, Ke Yang ${ }^{\mathrm{b}}$, Yu-Xiao Liu ${ }^{\mathrm{c}}$ \\ Institute of Theoretical Physics, Lanzhou University, Lanzhou 730000, People's Republic of China
}

Received: 24 June 2015 / Accepted: 6 July 2015 / Published online: 17 July 2015

(C) The Author(s) 2015. This article is published with open access at Springerlink.com

\section{Erratum to: Eur Phys J C (2015) 75:253 DOI 10.1140/epjc/s10052-015-3469-7}

In the published paper, there were two issues on the gravitational lensing that need to be corrected and clarified.

(i) The deflection angle $\alpha\left(r_{0}\right)$ of the photon (see Eqs. (42) and (43) in the published paper) for the most general static spherically symmetric metric $\mathrm{d} s^{2}=-A(r) \mathrm{d} t^{2}+B(r) \mathrm{d} r^{2}+$ $C(r)\left(\mathrm{d} \theta^{2}+\sin ^{2} \theta \mathrm{d} \phi^{2}\right)$ was in fact obtained by Virbhadra et al. [1] in the year 1998 .

$\alpha\left(r_{0}\right)=I\left(r_{0}\right)-\pi$,

$I\left(r_{0}\right)=2 \int_{r_{0}}^{\infty} \frac{\sqrt{B}}{\sqrt{C} \sqrt{\frac{C A_{0}}{C_{0} A}-1}} \mathrm{~d} r$.

In the same paper, they [1] also obtained impact parameter $J\left(r_{0}\right)$ of the deflected light:

$J\left(r_{0}\right)=r_{0} \sqrt{\frac{C\left(r_{0}\right)}{A\left(r_{0}\right)}}$

that we used in calculating impact parameter of photon sphere.

(ii) Photon sphere. Virbhadra and Ellis [2] and Claudel et al. [3] gave 2 different definitions of photon sphere for a general static spherically symmetric space-time and then they $[3,4]$ showed that both definitions give same result:
$\frac{C^{\prime}(r)}{C(r)}=\frac{A^{\prime}(r)}{A(r)}$,

which reduces to $A^{\prime} r=2 A$, and Eq. (44) of our published paper will be obtained.

Thus, in this erratum, we clarify that these authors derived such equations that we ignored stating in the published paper.

We are immensely grateful to K. S. Virbhadra for pointing out these to us.

Open Access This article is distributed under the terms of the Creative Commons Attribution 4.0 International License (http://creativecomm ons.org/licenses/by/4.0/), which permits unrestricted use, distribution, and reproduction in any medium, provided you give appropriate credit to the original author(s) and the source, provide a link to the Creative Commons license, and indicate if changes were made.

Funded by $\mathrm{SCOAP}^{3}$.

\section{References}

1. K.S. Virbhadra, D. Narasimha, S.M. Chitre, Astron. Astrophys. 337, $1(1998)$

2. K.S. Virbhadra, G.F.R. Ellis, Phys. Rev. D 62, 084003 (2000)

3. C.M. Claudel, K.S. Virbhadra, G.F.R. Ellis, J. Math. Phys. 42, 818 (2001)

4. K.S. Virbhadra, G.F.R. Ellis, Phys. Rev. D 65, 103004 (2002)

The online version of the original article can be found under doi:10.1140/epjc/s10052-015-3469-7.

\footnotetext{
a e-mail: weishw@1zu.edu.cn

b e-mail: yangke09@1zu.edu.cn

c e-mail: liuyx@1zu.edu.cn
} 